\title{
Electrospray deposition and direct patterning of polylactic acid nanofibrous microcapsules for tissue engineering
}

\author{
Masashi Ikeuchi • Ryosuke Tane • Koji Ikuta
}

Published online: 6 September 2011

(C) The Author(s) 2011. This article is published with open access at Springerlink.com

\begin{abstract}
Electrospun nanofibers composed of biodegradable polymers are attractive candidates for cell culture scaffolds in tissue engineering. Their fine-meshed structures, resembling natural extracellular matrices, effectively interact with cell surfaces and promote cell proliferation. The application of electrospinning, however, is limited to twodimensional (2D) or single tube-like scaffolds, and the fabrication of arbitrary three-dimensional (3D) scaffolds from electrospun nanofibers is still very difficult due to the fibers' continuous and entangled form. To address this issue, in this paper, we describe the use of phase-separation-assisted electrospray and electrostatic focusing to perform continuous direct 3D patterning of nanofibrous microcapsules of biodegradable polylactic acid (PLA). These microcapsules exhibit fiber-particle duality because they are composed of nanofibers suitable for cell attachment while also being easy to handle as particles for direct 3D patterning. By varying the flow rate of the polymer solution and the humidity of the electrospray
\end{abstract}

Electronic supplementary material The online version of this article (doi:10.1007/s10544-011-9583-x) contains supplementary material, which is available to authorized users.

M. Ikeuchi $(\bowtie) \cdot R$. Tane $\cdot$ K. Ikuta

Research Center for Advanced Science and Technology,

The University of Tokyo,

4-6-1 Komaba, Meguro-ku,

Tokyo 153-8904, Japan

e-mail: ikeuchi@micro.rcast.u-tokyo.ac.jp

\section{Ikeuchi}

PRESTO, Japan Science and Technology (JST),

4-1-8 Honcho, Kawaguchi,

Saitama 332-0012, Japan

K. Ikuta

Graduate School of Information Science and Technology,

The University of Tokyo,

7-3-1 Hongo, Bunkyo-ku,

Tokyo 113-8656, Japan atmosphere during electrospraying, the diameter of the microcapsule and its surface porosity can be controlled. The utility of the direct-patterning process is demonstrated by fabricating high-aspect-ratio microscaffolds and subsequent cell cultures. The nanofibrous and hollow structure of the microcapsules combined with the direct $3 \mathrm{D}$ patterning process offers a new approach for fabricating tailor-made scaffolds for regenerative medicine.

Keywords Microcapsule - Micropatterning ·

Microstructure $\cdot$ Porous material $\cdot$ Polylacticacid $\cdot$ Scaffold

\section{Introduction}

It is important that tissue engineering scaffolds include 3D interconnected spaces to facilitate diffusion of culture medium and cell interactions, and at the same time, should also provide suitable surface morphology for cell attachment and proliferation (Causaa et al. 2007). Nanofibers of biodegradable polymers produced by electrospinning have been found to be a potential material to fulfill these purposes ( $\mathrm{Li}$ et al. 2002; Huang et al. 2003; Teo and Ramakrishna 2006; Chung and Park 2007; Sill and Recum 2008). In electrospinning, a polymer solution is ejected from a charged nozzle toward a target electrode plate of opposite polarity. After being ejected from the nozzle tip, the charged solution jet elongates and randomly splits into many thinner fibers due to electrostatic repulsion and bending instability. Thus, a mesh of nanofibers is accumulated on the target electrode. To culture cells in a defined geometry, the patterning of electrospun nanofibers has been investigated using gas flow (Zhou et al. 2007), by applying a magnetic force to electrospun nanofibers impregnated with magnetic particles (Yang et al. 2007) or by patterning conductive and nonconductive areas on a target electrode plate $(\mathrm{Li}$ et al. 
2003, 2005). The use of these methods, however, limits the patterning to only local alignment of the nanofibers, and does not enable the fabrication of $3 \mathrm{D}$ structures due to the continuity of highly branched electrospun nanofibers. Another method for fabricating scaffolds from electrospun nanofibers is to electrospin on a rotating cylindrical target electrode (Xiang et al. 2004; Katta et al. 2004). The nanofibers that are spun around the electrode form a tubelike scaffold, which can be used for blood vessel regeneration. However, this process is only applicable to large tubelike structures. To overcome this drawback, it is highly desirable to develop a new process to pattern arbitrary $2 \mathrm{D}$ and $3 \mathrm{D}$ structures of biodegradable nanofibers.

In electrospinning, the viscosity of the polymer solution affects the morphology of the final product (Fong et al. 1999). By decreasing the viscosity of the polymer solution, the polymer solution jet is gradually thinned and the diameter of the fiber becomes nonuniform, which results in a beaded fiber - an undesired product in electrospinning. In a process called electrospray, the viscosity is further decreased so that the polymer jet breaks up into tiny droplets, and microparticles are obtained (Jaworek 2007). Simultaneously, the ambient humidity and volatility of the solvent affect the surface morphology of electrospun products. Under high ambient humidity or with highly volatile solvents, submicron-sized pores are formed on the surface of electrospun fibers due to phase separation or the breath-figure effect during electrospinning (Bognitzki et al. 2001; Megelski et al. 2002; Casper et al. 2004; Qi et al. 2009; Han et al. 2005).

In the present study, we aim to combine the electrospray technique and the phase separation phenomenon to obtain microcapsules with a nanofibrous surface. The novelty of the structure lies in its fibre-particle duality. The nanofibrous surface of the microcapsule is expected to enhance cell attachment, similar to electrospun nanofibres. At the same time, the microcapsule can be treated as a particle in contrast to higly branched electrospun nanofibers, allowing direct patterning. Our goal is to produce these nanofibrous microcapsules, and stack them three-dimensionally to fabricate a cell culture scaffold with high porosity, interconnectivity and good cell attaching characteristics.

\section{Materials and methods}

\subsection{Electrospray of poly-lacticacid (PLA)}

PLA (PURASORB PL, viscosity: $4.52 \mathrm{dl} / \mathrm{g}$ ) was purchased from Purac Biomaterials (Japan). Special grade ethanol and chloroform were purchased from Wako Pure Chemical Industries (Japan) and used without further purification. To prepare the electrospraying solution, firstly PLA was dissolved overnight in chloroform. Then the secondary solvent ethanol was added to the solution. The concentration of PLA was increased from $0.5 \%$ to $2.0 \%$. The concentration of ethanol was increased from $0 \%$ to $7.5 \%$. Concentrations of the solutions are described by the weight percentage of the material to the total weight of the solution.

The electrospraying apparatus was basically composed of a grounded target electrode made of an aluminium plate, and a 19G stainless steel syringe needle (Azone, Japan) placed $178 \mathrm{~mm}$ above the target electrode by an insulating holder. $20 \mathrm{kV}$ was applied between the syringe needle and the target electrode for electrospraying. The syringe needle and a motorized injection pump were connected with a Teflon tube, and the solution was supplied to the syringe needle. The flow rate was increased from $40 \mu \mathrm{l} / \mathrm{min}$ to $120 \mu \mathrm{l} / \mathrm{min}$. The electrodes were built inside a closed chamber, and both temperature and humidity within the chamber were monitored and kept at designated values by a heater and a humidifier. The humidity was increased from $50 \%$ to $90 \%$.

To focus the microcapsules on the target electrode, an electrostatic lens was inserted between the syringe needle and the target electrode, installed $15 \mathrm{~mm}$ above the target electrode. The lens was a cylindrical cup (height: $150 \mathrm{~mm}$, inner diameter: $94 \mathrm{~mm}$ ) made of aluminium with a small opening (diameter: $20 \mathrm{~mm}$ ) at the bottom. $15 \mathrm{kV}$ was applied between the lens and the target electrode for focusing. To pattern the microcapsules, the target electrode was mounted on a computer controlled $\mathrm{X}-\mathrm{Y}-\mathrm{Z}$ stepping motor stage (Chuo Seiki, Japan). The entire setup of the system is shown in Fig. 1.

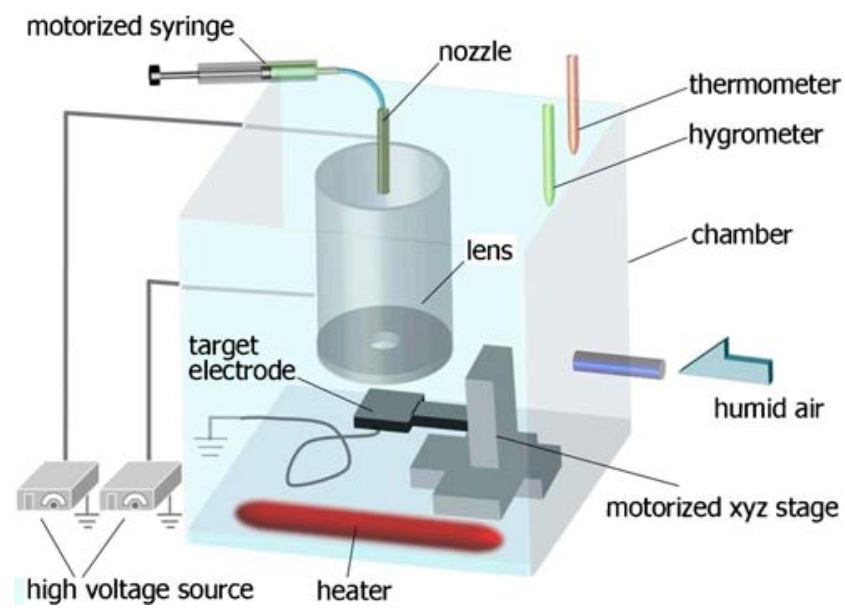

Fig. 1 Schematic of the direct 3D patterning system of nanofibrous microcapsules. The entire process is performed in a closed temperature- and humidity-controlled chamber. The polymer solution is supplied to the high-voltage nozzle by a motorised syringe, and the grounded target substrate is mounted onto a computer-controlled $X-Y-Z$ stage. The electrosprayed nanofibrous microcapsules are focused at the bottom aperture of the lens, and arbitrary 3D patterns can be produced in a single step by moving the stage 


\subsection{Measurement of the capsule}

Conventional volumetric measurements of porosity are not appropriate for this material because there are pores and cavities in multiple levels of scale-surface pore, capsule core and cavity between microcapsules. By volumetric measurements, only the mean value of the porosity of multiple levels of scale is obtained. Therefore, visual inspection is the most suitable method to measure the surface porosity of the nanofibrous microcapsule. The surface porosity was measured by visual inspection software (Vision Assistant, National Instruments). Microcapsules were observed using scanning electron microscope (SEM, JEOL) at 4000 magnification, and the images were saved as bitmap files at 1,280 $\times 960$ pixels resolution. Each image was initially binarized by setting a threshold at the dip between the two peaks of the brightness histogram so that the pores (dark area) and the polymer skeleton (bright area) were distinguished. Next, the ratio of black area to white area was calculated. The surface porosity was obtained by averaging this ratio over ten samples. The diameter of microcapsules was also measured using the same software. First, the outline of a microcapsule was obtained using the edge-detection function, and then, the edge was approximated as a circle to obtain the diameter of the capsule. The final diameter value was obtained by averaging over ten samples.

\subsection{Cell culture}

Stacked nanofibrous microcapsules were placed on the bottom of tissue culture plates (12well plate, IWAKI, Japan) and were sterilized with UV irradiation for $2 \mathrm{~h}$. They were soaked in PBS, and air bubbles trapped inside the nanofibrous microcapsules were degassed in vacuo. Control experiments were carried out using normal poly-styrene tissue culture plates (12well plate, IWAKI, Japan). HepG2 cell was seeded at a density of $1.06 \times 10^{3}$ cells $/ \mathrm{mm}^{2}$. The cells were incubated in a humidified atmosphere of $95 \%$ air, $5 \% \mathrm{CO}_{2}$ at $37^{\circ} \mathrm{C}$. Since the sheet of nanofibrous microcapsules were partly transparent in liquid medium, the number of cell was counted under optical microscope at $24 \mathrm{~h}, 48 \mathrm{~h}$ and $72 \mathrm{~h}$.

\subsection{MTT assay}

MTT assay was conducted after culturing for $120 \mathrm{~h}$. After discarding culture medium, cells were gently washed with PBS. Then new culture medium containing $100 \mu \mathrm{l}$ MTT $(0.5 \mathrm{mg} / \mathrm{ml})$ was added to each culture well and incubated for $4 \mathrm{~h}$. The MTT was allowed to crystalize to formazan pigment by metabolic active cells. Finally the culture medium was removed and dimethyl sulphoxide (DMSO) was added. The absorbance of the DMSO solution at
$570 \mathrm{~nm}$ was measured by an absorbance spectrometer (BioSpec 1600, Shimadzu, Japan).

\subsection{Scanning electron microscopy}

PBS, t-butanol and 10\% glutaraldehyde aqueous solution were purchased from Wako Pure Chemical Industries (Japan) and used without further purification. The cells cultured for $48 \mathrm{~h}$ on the nanofibrous microcapsules were fixed with $2 \%$ glutaraldehyde/PBS for $12 \mathrm{~h}$. First, the fixing solution was replaced to ethanol with ascending series of ethanol/water solution, and then completely replaced with t-butanol with ascending series of t-butanol/ethanol solution. The sample in t-butanol was freeze-dried at $-20^{\circ} \mathrm{C}$ and sputter-coated with Au using an ion sputter (Hitachi High-technologies, Japan). The sample was observed using a SEM (JEOL, Japan) under $20 \mathrm{kV}$.

\section{Results and discussion}

\subsection{Formation of nanofibrous microcapsules}

We investigated electrospraying of a PLA-chloroform-ethanol solution using chloroform as a solvent and ethanol as a nonsolvent. Nanofibers were formed with the solution containing 2\% PLA and $20 \%$ ethanol at a flow rate of $40 \mu \mathrm{l} /$ min (Fig. 2(a)). The diameter of the nanofiber was about $200 \mathrm{~nm}$. Beaded nanofibers were formed by decreasing the concentration of PLA to $1.5 \%$ while keeping the ethanol concentration at $20 \%$ under the same flow rate (Fig. 2(b)). By decreasing both the concentration of PLA and ethanol to $1 \%$ and $5 \%$ respectively, microcapsules were formed with a surface composed of a mesh of nanofibers (Fig. 3(a)). The diameter of the nanofiber was $100 \mathrm{~nm}$ to $200 \mathrm{~nm}$, which was similar to that of the electrospun nanofibers (Fig. 2(a)), and formed a thin shell of the capsule with penetrating pores (Fig. 3(b)). The possible mechanism of nanofibrous microcapsule formation is as follows: 1) the polymer solution jet that is electrosprayed from the nozzle breaks up into tiny droplets because of bending instability and electrostatic repulsion; 2) the polymer solution droplet expands to a microspherical shell because of electrostatic repulsion; 3) the droplet is subjected to high ambient humidity conditions, and water vapour is absorbed onto the surface of the droplet, causing phase separation (Zheng et al. 2006) between the nonsolvent and solvent phases; and 4) the water and the solvent rapidly evaporate, leaving the polymer to form a nanofibrous microcapsule. The second and third steps can occur simultaneously (Fig. 3(c)).

Although reports on the electrospray formation of microscale beads and cup-like structures with penetrating micropores on their surface exist (Liu and Kumar 2005; Liu 
Fig. 2 SEM image of electrospun products of poly-lacticacid; (a) nanofibers (b) beaded nanofibers. Nanofibers were formed with the solution containing $2 \%$ PLA and $20 \%$ ethanol. Beaded nanofibers were formed with the solution containing 1\% PLA and $20 \%$ ethanol. The scale bars are $50 \mu \mathrm{m}$
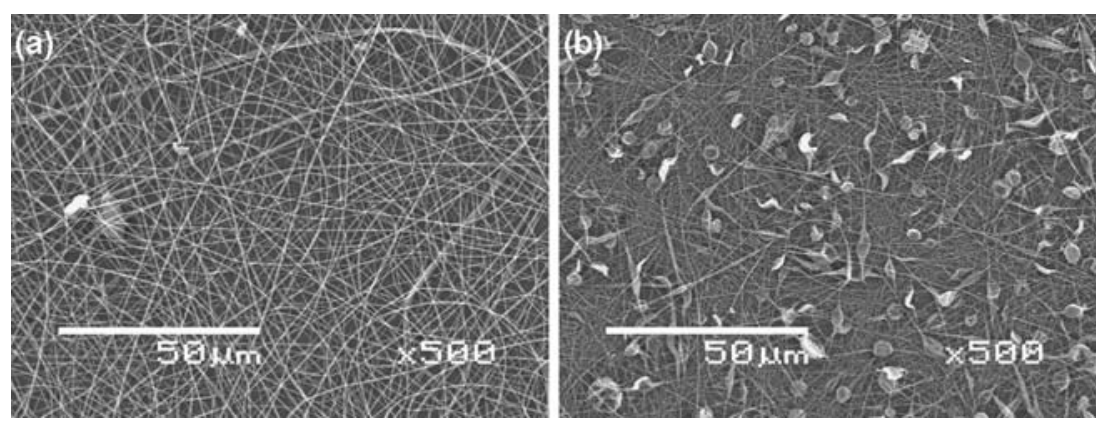

et al. 2008; Wu and Clark 2007; Eda and Shivkumar 2006), there are no reports on the formation of microcapsules having nanofibrous meshed surfaces. The morphology of an electrosprayed product is very sensitive to various parameters including solution composition, ambient humidity, flow rate, applied voltage and nozzle diameter. Among these parameters, we find that the key to highly stabilized formation of nanofibrous microcapsules is the combination of the usage of chloroform-ethanol solvent and high ambient humidity.

\subsection{Effects of electrospray parameters}

To investigate the effect of solution composition, the ratio of ethanol as a nonsolvent was increased from $0 \%$ to $7.5 \%$ while keeping the concentration of PLA at $1 \%$ under a flow rate of
$40 \mu \mathrm{l} / \mathrm{min}$. The solution containing $5 \%$ of ethanol allows for a stable and uniform formation (Fig. 4(a)) of spherical microcapsules as compared to the formation obtained with less ethanol ratio of $0 \%$ and $2.5 \%$ (Fig. 4(b and c)). The microcapsule morphology appears to be determined by competition between phase separation and solvent evaporation. To achieve a nanofibrous meshed surface upon a uniformly expanded spherical shell, phase separation should be complete before solvent evaporation finishes. Based on our observations, by adding ethanol, the electrosprayed jet splitting point is brought closer to the jet nozzle. Because water is miscible with ethanol, the addition of ethanol causes the onset of phase separation to occur sooner, resulting in increased jet instability (Dayal et al. 2007; Pai et al. 2009). Thus, the time of flight is sufficient for the
Fig. 3 Formation of nanofibrous microcapsules by phaseseparation-assisted electrospray; (a) Overview SEM image of the microcapsules. Nanofibrous microcapsules were continuously and stably produced under appropriate conditions. The scale bar is $50 \mu \mathrm{m}$. (b) Magnified SEM image of a microcapsule. The shell of the microcapsule consists of meshed nanofibers $100 \mathrm{~nm}$ in diameter. The scale bar is $5 \mu \mathrm{m}$. (c) Schematic of the formation mechanism of nanofibrous microcapsules
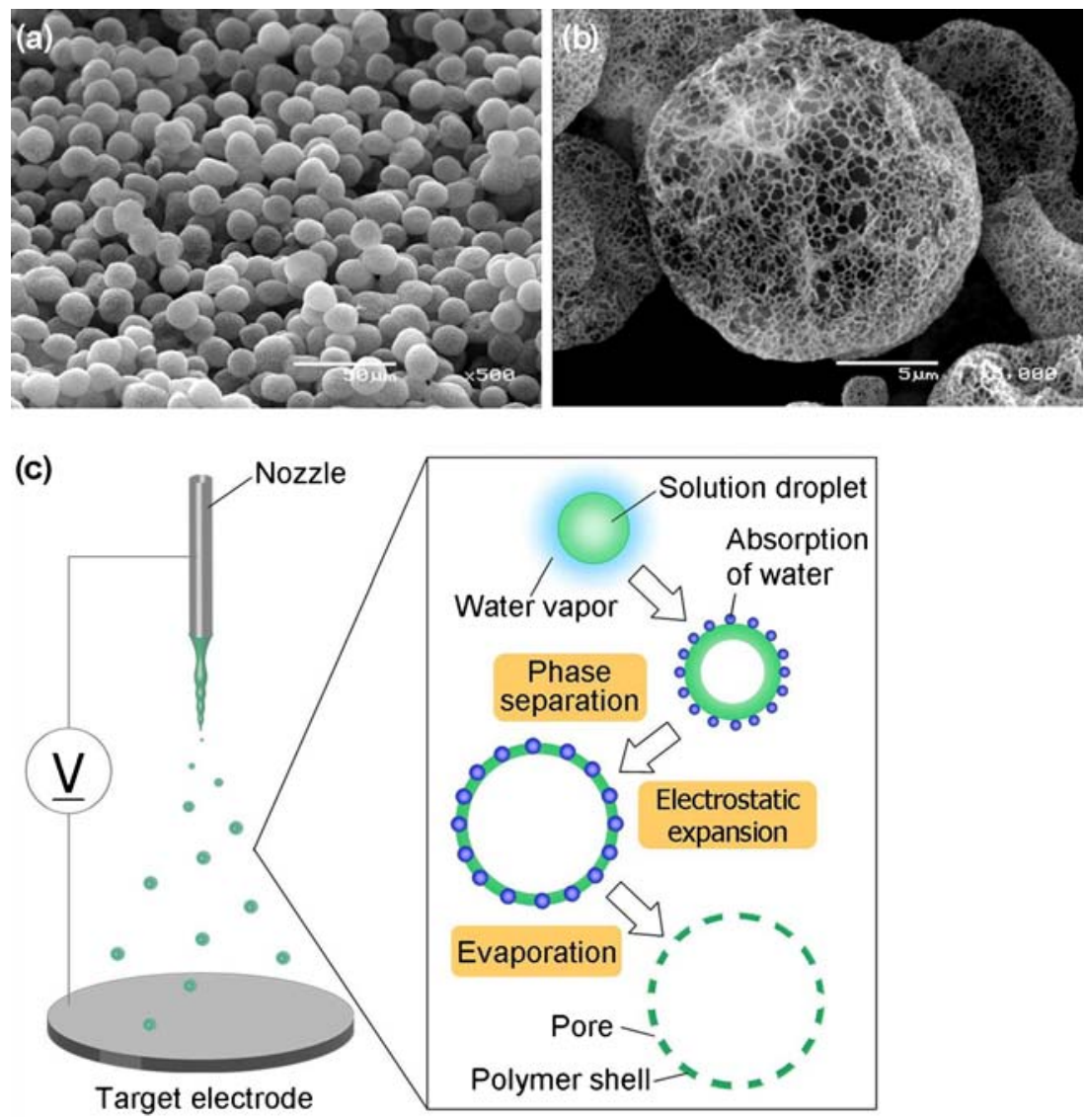
Fig. 4 SEM image of the electrosprayed products of $1 \mathrm{wt}$ $\%$ PLA solution. The ethanol: chloroform ratio in the solvent was varied; (a) chloroform containing $5.0 \%$ ethanol, (b) $100 \%$ chloroform, (c) chloroform containing $2.5 \%$ ethanol, (d) chloroform containing $7.5 \%$ ethanol
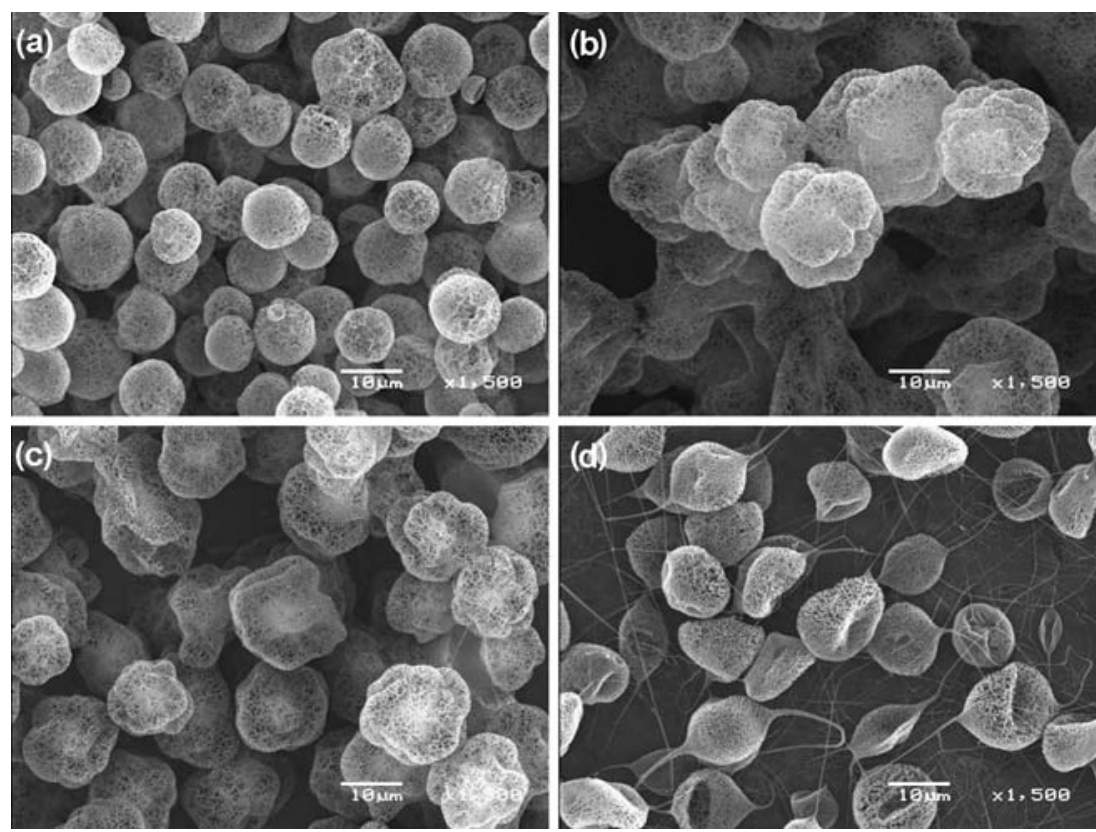

solution droplets to complete phase separation before solvent evaporation. In addition, excessive ethanol resulted in nanofiber or beaded-nanofiber formation rather than the formation of nanofibrous microcapsules with the solution containing 7.5\% ethanol (Fig. 4(d)). This probably occurs because the PLA concentration in the solvent phase increases after phase separation into the PLA-chloroform and ethanolwater phases, which causes the viscosity of the solvent phase to increase. For following experiments, we experimentally determined the appropriate ratio of PLA and ethanol in chloroform to $1 \%$ and $5 \%$ respectively.

The effect of ambient humidity on the surface porosity of the nanofibrous surface was investigated with the solution containing 1\% PLA and 5\% ethanol under a flow rate of $60 \mu \mathrm{l} /$ min. By changing the ambient humidity from $50 \%$ to $90 \%$, the surface porosity was increased from $24 \%$ to $56 \%$ almost in proportional to the ambient humidity (Fig. 5). This occurs because the amount of water absorbed onto the droplet surface increases with humidity, which leaves more pores on the final product through vapour-induced phase separation over larger areas of the shell. At lower humidity, microcapsules with no pores are produced, because not enough water vapour is absorbed to induce phase separation. Most microcapsules without pores are flattened or wrinkled, most likely because the electrostatic repulsion that supports the shell structure against compressive stress by atmospheric pressure diminishes after the microcapsule reaches the target electrode. The nanofibrous microcapsules maintain their shape, however, because penetrating micropores keep the inner and outer pressure of the microcapsule in equilibrium.

In addition, the relationship between the flow rate and the diameter of the nanofibrous capsule was investigated with the solution containing 1\% PLA and 5\% ethanol. The diameter of the microcapsule was tunable from $\phi=14 \mu \mathrm{m}$ to $\phi=19 \mu \mathrm{m}$ by adjusting the solution flow rate from $40 \mu \mathrm{l} /$ $\min$ to $120 \mu \mathrm{l} / \mathrm{min}$, and the diameter increased with the flow rate (Fig. 6). A higher flow rate appears to cause an increase in the initial diameter of the electrosprayed
Fig. 5 Relation between the surface porosity of microcapsules and the ambient humidity. The porosity increases with humidity because the water vapour absorption increases. The error bar shows one standard deviation. The inset shows SEM images of microcapsules obtained under each humidity conditions
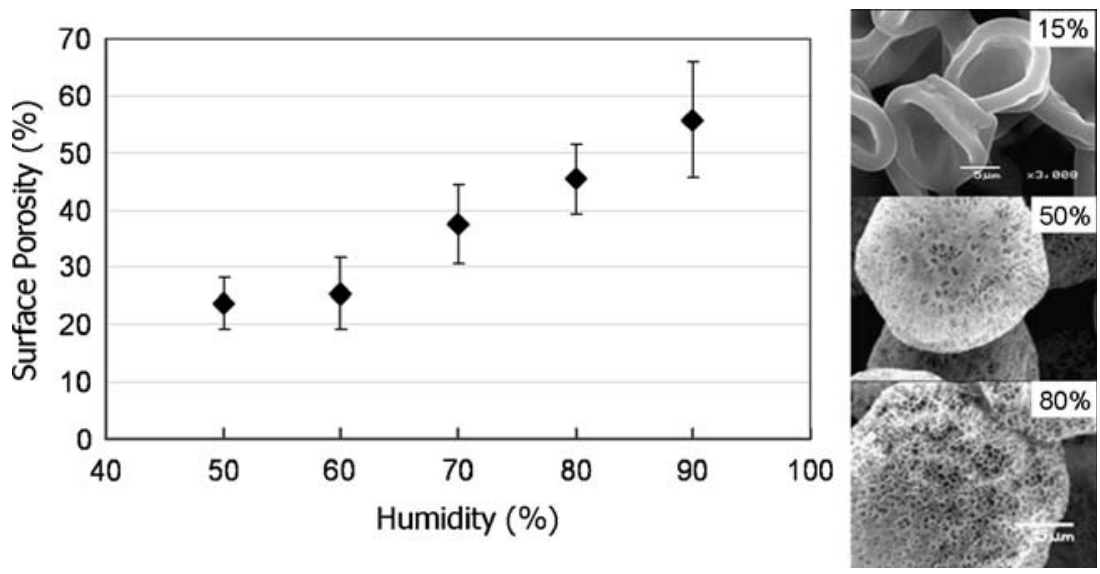
Fig. 6 Relation between the microcapsule diameter and the solution flow rate through the nozzle. The diameter increases with the flow rate. The error bar shows one standard deviation. The inset shows SEM images of microcapsules obtained under each humidity conditions
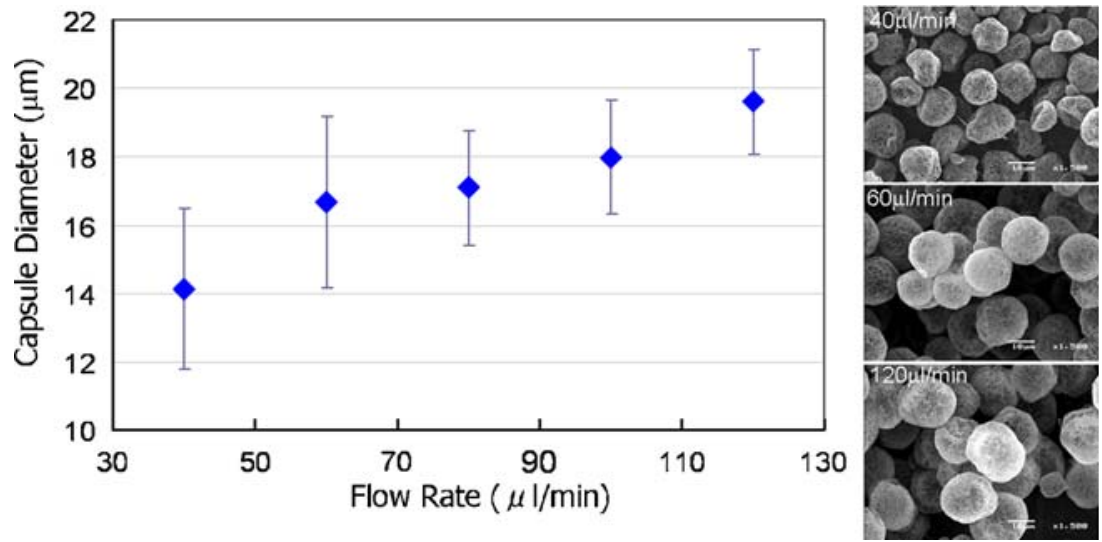

solution jet from the nozzle, resulting in the formation of larger initial droplets due to surface instability and larger microcapsules at the end.

\subsection{Direct patterning of the nanofibrous microcapsule}

To create $3 \mathrm{D}$ tissue-engineering scaffolds from nanofibrous microcapsules, the distance between the nozzle and the target electrode was determined so that the time of flight of the microcapsules would be sufficient for them to expand and, at the same time, the microcapsules could reach the electrode before the solvent completely evaporates. In this way, the microcapsules deposited on the electrode are interconnected to provide a 200- $\mu$ m-high stacked-sheet structure (Fig. 7(a)). The sheet consists entirely of uniform capsules from top to bottom and is sufficiently mechani-
Fig. 7 Fabrication of a 3D scaffold with nanofibrous microcapsules; (a) SEM image of a sheet made of accumulated nanofibrous microcapsules. The inset shows the entire sheet handled with tweezers. The scale bar is $100 \mu \mathrm{m}$ (inset scale bar is $10 \mathrm{~mm}$ ). (b) SEM image of a pillar made of nanofibrous microcapsules focused by an electrostatic lens. The scale bar is $200 \mu \mathrm{m}$. (c) Magnified SEM image of the middle part of the pillar. The scale bar is $50 \mu \mathrm{m}$. (d) Twodimensional line patterns of nanofibrous microcapsules. The inset shows an SEM image of a segment of the line. The scale bar is $10 \mathrm{~mm}$ (inset scale bar is $1 \mathrm{~mm}$ ). (e) $2 \mathrm{~mm}$ square microwell wiht a height of $500 \mu \mathrm{m}$ fabricated by direct deposition and patterning of nanofibrous microcapsules. The scale bar is $1 \mathrm{~mm}$
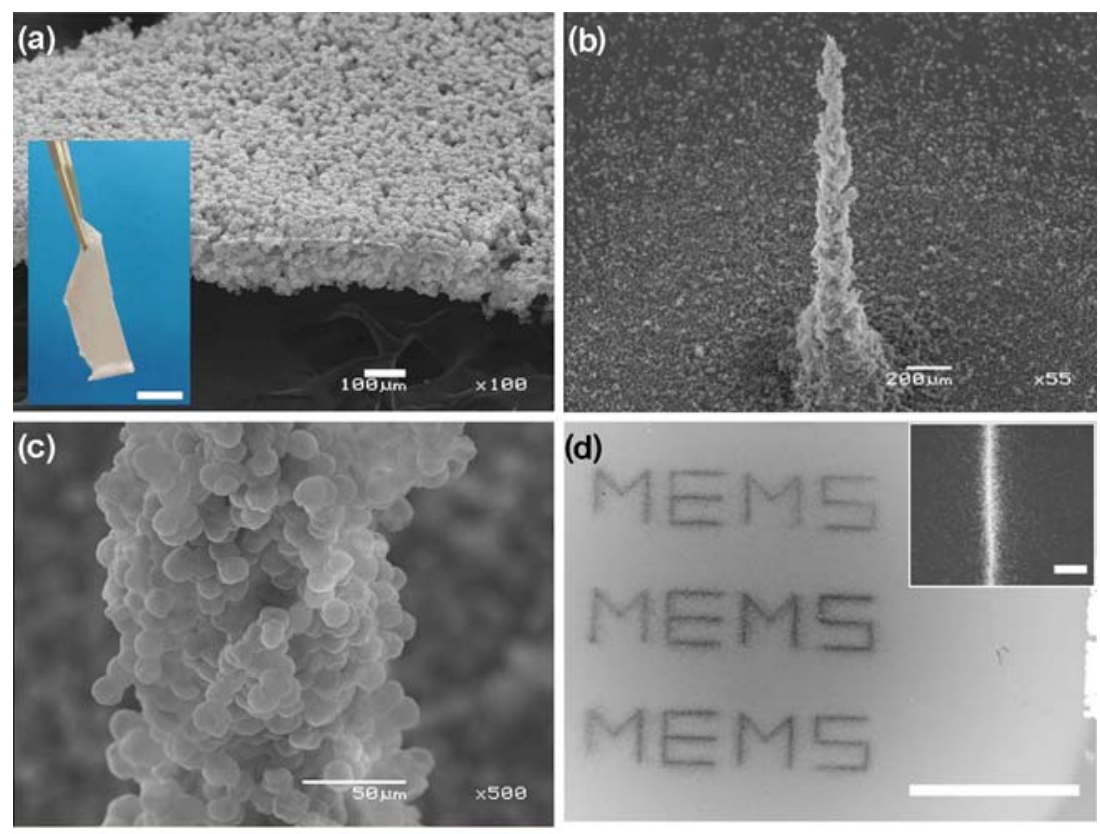

(e)

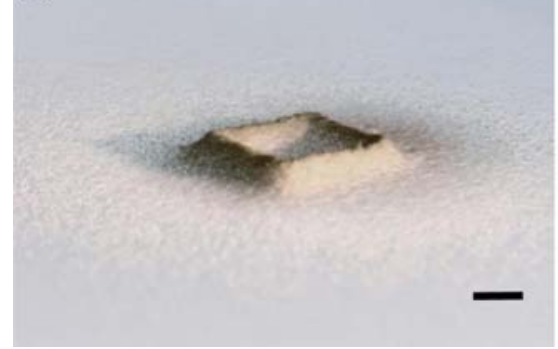


cally stable to be manipulated with tweezers (Fig. 7(a) inset). The structure thickness can be easily controlled by changing the electrospray duration.

Next, we introduced an electrostatic lens placed in the path of the electrospray and concentric with the ejection nozzle to focus the electrosprayed nanofibrous microcapsules. Since the electrosprayed nanofibrous microcapsules were positively charged, they were subjected to repulsive force from the wall of the cylindrical electrostatic lens and focused at the bottom aperture of the lens. This setup can achieve continuous microcapsule formation and simultaneous direct 3D patterning of microcapsules on arbitrary substrates by moving the stage.

To verify the formation and simultaneous electrostatic focusing of the nanofibrous microcapsules, they were focused to a single spot on a glass substrate placed on the target electrode (Fig. 7(b and c)). The height of the resulting microcapsule pillar increases with electrospray duration, whereas the bottom width remains constant because of electrostatic focusing. The resulting height of the pillar was $2,000 \mu \mathrm{m}$, and the diameter of the pillar was $500 \mu \mathrm{m}$ at the bottom and $200 \mu \mathrm{m}$ on average. By moving the stage, lines of nanofibrous microcapsules

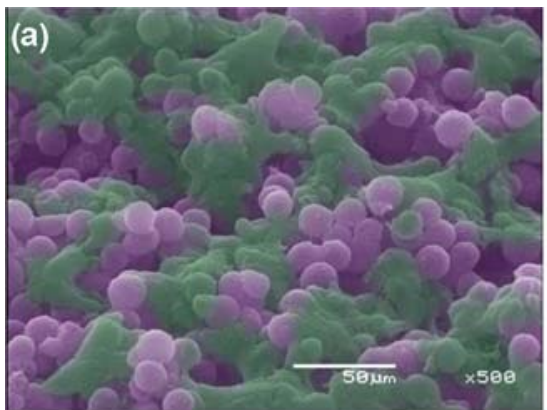

(c)

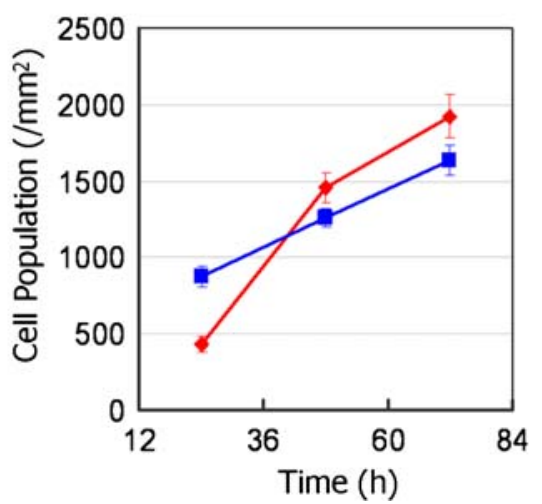

Fig. 8 HepG2 cell culture on a sheet of nanofibrous microcapsules; (a) SEM image of cells (green) cultivated for $48 \mathrm{~h}$ on a sheet of nanofibrous microcapsules (purple) with manual pseudo-colour. The scale bar is $50 \mu \mathrm{m}$. (b) Magnified SEM image of the interface between a cell (green) and a nanofibrous microcapsule (purple) with manual pseudo-colour. The cell tightly adheres to the nanofibers of the microcapsule. The scale bar is $5 \mu \mathrm{m}$. (c) Cell-proliferation rate plotted could be drawn in arbitrary geometries on the glass substrate (Fig. 7(d), Supplementary Movie). The resolution of the line patterning is $500 \mu \mathrm{m}$ in width. In addition, by moving all three axes, a $2 \mathrm{~mm}$ square microwell with a height of $500 \mu \mathrm{m}$ was patterned on the substrate (Fig. 7(e)). In contrast with the previously reported method for patterning electrospun nanofibers that involves fabricating 3D columnar collectors on the target substrate (Zhang and Chang 2008), the direct-patterning method developed herein is highly flexible (note that conventional electrospun nanofibers are difficult to pattern directly in this way because of their continuous form). Thus, it is easy to modify the pattern, making this method suitable for fabricating tailor-made products for medical applications.

Although the mechanical strength of the structure composed of nanofibrous microcapsules remains to be measured, the structure had enough strength to be manipulated with tweezers for cell seeding and culture. The structure is intended to be used as a scaffold of in vitro cell culture, and the regenerated tissue is expected to be transplanted to a patient after degradation of the scaffold. Therefore, the mechanical strength of the structure itself is enough for our purpose.

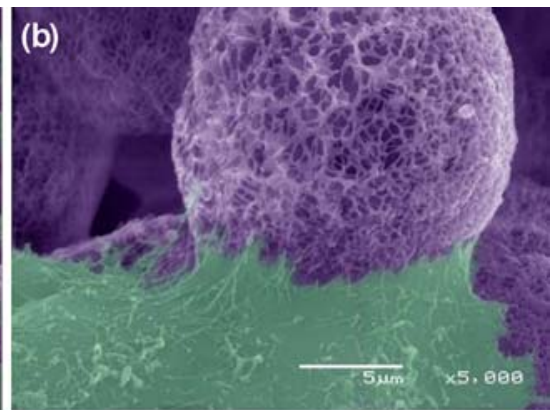

(d)

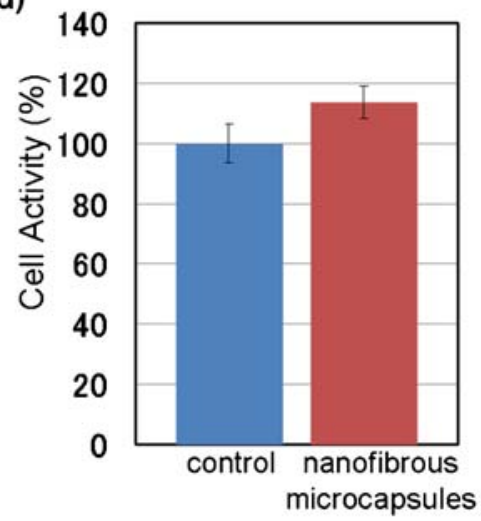

versus culture time in hours. After $48 \mathrm{~h}$, the cells cultured on the sheet of nanofibrous microcapsules (red) show proliferation equal to or surpassing that of the cells cultured on tissue-culture polystyrene plates (blue). (d) MTT assay results after $120 \mathrm{~h}$ of culture on the sheets of nanofibrous microcapsules (red) and the normal cell culture plates (blue). The cell activity on the normal culture plate was standardized as $100 \%$ 


\subsection{Cyto-compatibility of the nanofibrous microcapsules}

To demonstrate a tissue-engineering application of the nanofibrous microcapsules, we cultured human hepatocyte cells on the 3D structure composed of nanofibrous microcapsules. Although plain PLA film has poor characteristics for cell culture due to its hydrophobic characteristics, we achieved good cell adhesion and proliferation on the sheet of nanofibrous microcapsules without any specific coating (Fig. 8(a)). From the magnified view, the edge of the cell membrane seemed to highly attached and adhered to the nanofibers of the shell (Fig. 8(b)). After $48 \mathrm{~h}$ of cultivation, the cell-proliferation rate either equalled or surpassed that of cells cultured on normal tissue culturing polystyrene plates (Fig. 8(c)). The results of the MTT assay also showed that the cell activity upon the developed sheet composed of nanofibrous microcapsules was approximately $114 \%$ in comparison with normal cell culture plates shown as $100 \%$ (Fig. 8(d)). It can be speculated that the nanofibrous surface promoted initial cell attachment resulting in increased cell activity, and the hollow microcapsule structure allowed nutrients and gases to circulate within the structures, making them suitable for cell proliferation. However, whether the medium and gas regulating hollow structure had had a positive effect on cell proliferation after cell seeding is unknown and yet to be investigated.

\section{Conclusions}

We have developed a stable production process of polylactic acid microcapsules with nanofibrous shells by successfully combining electrospraying techniques with the phase separation phenomenon. The shape, surface porosity and the diameter of the nanofibrous microcapsules were found to be tuneable by adjusting the solution mixture, ambient humidity and flow rate. Good cell proliferation and adhesion were verified on the nanofibrous microcapsule formed sheet by in vitro cell culture. The biodegradability and the long-term interaction of the nanofibrous microcapsules with cells should be investigated for further application in tissue regeneration.

In addition, by directly focusing the produced microcapsules to a single spot on a target plate and by moving the target plate on a motorized stage, a novel fabrication process of simple 2D and 3D structures composed of nanofibrous microcapsules were demonstrated. Although packing in vertical direction is still quite random and more precise control of $3 \mathrm{D}$ deposition is remained to be solved, the nanofibrous and hollow structure of the microcapsule combined with the direct $3 \mathrm{D}$ patterning process will open up a novel type of custom-made scaffolds for tissue engineering.
Acknowledgements The authors thank Dr. Akira Yamada, Muneaki Fukuoka and Kuniho Kasugai for their assistance in starting the project.

Open Access This article is distributed under the terms of the Creative Commons Attribution Noncommercial License which permits any noncommercial use, distribution, and reproduction in any medium, provided the original author(s) and source are credited.

\section{References}

M. Bognitzki, W. Czado, T. Frese, A. Schaper, M. Hellwig, M. Steinhart, A. Greiner, J.H. Wendorff, Nanostructured fibers via electrospinning. Adv. Mater. 13(1), 70-72 (2001)

C.L. Casper, J.S. Stephens, N.G. Tassi, D. Chase, J.F. Rabolt, Controlling surface morphology of electrospun polystyrene fibers: Effect of humidity and molecular weight in the electrospinning process. Macromolecules 37(2), 573-578 (2004)

F. Causaa, P.A. Nettib, L. Ambrosiob, A multi-functional scaffold for tissue regeneration: The need to engineer a tissue analogue. Biomaterials 28, 5093-5099 (2007)

H.J. Chung, T.G. Park, Surface engineered and drug releasing prefabricated scaffolds for tissue engineering. Adv. Drug Deliv. Rev. 59(4-5), 249-262 (2007)

P. Dayal, J. Liu, S. Kumar, T. Kyu, Experimental and theoretical investigations of porous structure formation in electrospun fibers. Macromolecules 40(21), 7689-7694 (2007)

G. Eda, S. Shivkumar, Bead structure variations during electrospinning of polystyrene. J. Mater. Sci. 41(17), 5704-5708 (2006)

H. Fong, I. Chun, D.H. Reneker, Beaded nanofibers formed during electrospinning. Polymer 40, 4585-4592 (1999)

S.O. Han, W.K. Son, J.H. Youk, T.S. Lee, W.H. Park, Ultrafine porous fibers electrospun from cellulose triacetate. Mater. Lett. 59(24 25), 2998-3001 (2005)

Z.M. Huang, Y.Z. Zhang, M. Kotaki, S. Ramakrishna, A review on polymer nanofibers by electrospinning and their applications in nanocomposites. Compos. Sci. Technol. 63(15), 2223-2253 (2003)

A. Jaworek, Micro- and nanoparticle production by electrospraying. Powder Technol. 176(1), 18-35 (2007)

P. Katta, M. Alessandro, R.D. Ramsier, G.G. Chase, Continuous electrospinning of aligned polymer nanofibers onto a wire drum collector. Nano Lett. 4(11), 2215-2218 (2004)

W.J. Li, C.T. Laurencin, E.J. Caterson, R.S. Tuan, F.K. Ko, Electrospun nanofibrous structure: A novel scaffold for tissue engineering. J. Biomed. Mater. Res. A 60(4), 613-621 (2002)

D. Li, Y. Wang, Y. Xia, Electrospinning of polymeric and ceramic nanofibers as uniaxially aligned arrays. Nano Lett. 3(8), 1167 $1171(2003)$

D. Li, G. Ouyang, J.T. McCann, Y. Xia, Collecting electrospun nanofibers with patterned electrodes. Nano Lett. 5(5), 913-916 (2005)

J. Liu, S. Kumar, Microscopic polymer cups by electrospinning. Polymer 46(10), 3211-3214 (2005)

J. Liu, A. Rasheed, H. Dong, W.W. Carr, M.D. Dadmun, S. Kumar, Electrospun micro- and nanostructured polymer particles. Macromol. Chem. Phys. 209(23), 2390-2398 (2008)

S. Megelski, J.S. Stephens, B. Chase, J.F. Rabolt, Micro- and nanostructured surface morphology on electrospun polymer fibers. Macromolecules 35(22), 8456-8466 (2002)

C.L. Pai, M.C. Boyce, G.C. Rutledge, Morphology of porous and wrinkled fibers of polystyrene electrospun from dimethylformamide. Macromolecules 42(6), 2102-2114 (2009) 
Z. Qi, H. Yu, Y. Chen, M. Zhu, Highly porous fibers prepared by electrospinning a ternary system of nonsolvent/solvent/poly (1-lactic acid). Mater. Lett. 63(3-4), 415-418 (2009)

T.J. Sill, H.A. Recum, Electrospinning: Applications in drug delivery and tissue engineering. Biomaterials 29(13), 1989-2006 (2008)

W.E. Teo, S. Ramakrishna, A review on electrospinning design and nanofibre assemblies. Nanotechnology 17, R89-R106 (2006)

Y. Wu, R.L. Clark, Controllable porous polymer particles generated by electrospraying. J. Colloid Interface Sci. 310(2), 529-535 (2007)

R.Z. Xiang, C.C. Chang, W.S. Fann, Electrospinning of continuous aligned polymer fibers. Appl. Phys. Lett. 84(7), 1222-1224 (2004)
D. Yang, B. Lu, Y. Zhao, X. Jiang, Fabrication of aligned fibrous arrays by magnetic electrospinning. Adv. Mater. 19, 3702-3706 (2007)

D. Zhang, J. Chang, Electrospinning of three-dimensional nanofibrous tubes with controllable architectures. Nano Lett. 8(10), 32833287 (2008)

J. Zheng, A. He, J. Li, J. Xu, C.C. Han, Studies on the controlled morphology and wettability of polystyrene surfaces by electrospinning or electrospraying. Polymer 47(20), 7095-7102 (2006)

W. Zhou, Z. Li, Q. Zhang, Y. Liu, F. Wei, G. Luo, Gas flow-assisted alignment of super long electrospun nanofibers. J. Nanosci. Nanotechnol. 7, 2667-2673 (2007) 